\title{
Detecting the Attenuation of Blazar Gamma-ray Emission by Extragalactic Background Light with GLAST
}

\author{
Andrew Chen \\ chen@mi.iasf.cnr.it \\ Consorzio Interuniversitario per la Fiscia Spaziale, \\ Torino, Italy 10133 \\ and \\ Istituto di Astrofisica Spaziale e Fisica Cosmica, Sezione di Milano, \\ Milano, Italy 20133 \\ Luis C. Reyes \\ University of Maryland at College Park, \\ College Park, MD 20742 \\ and \\ Laboratory for High Energy Astrophysics, NASA/Goddard Space Flight Center, \\ Greenbelt, MD 20771 \\ Steven Ritz \\ Laboratory for High Energy Astrophysics, NASA/Goddard Space Flight Center, \\ Greenbelt, MD 20771
}

\begin{abstract}
Gamma rays with energy above $10 \mathrm{GeV}$ interact with optical-UV photons resulting in pair production. Therefore, a large sample of high redshift sources of these gamma rays can be used to probe the extragalactic background starlight (EBL) by examining the redshift dependence of the attenuation of the flux above $10 \mathrm{GeV}$. GLAST, the next generation high-energy gamma-ray telescope, will have the unique capability to detect thousands of gamma-ray blazars to redshifts of at least $z=4$, with sufficient angular resolution to allow identification of a large fraction of their optical counterparts. By combining established models of the gamma-ray blazar luminosity function, two different calculations of the high
\end{abstract}


energy gamma-ray opacity due to EBL absorption, and the expected GLAST instrument performance to produce simulated fluxes and redshifts for the blazars that GLAST would detect, we demonstrate that these gamma-ray blazars have the potential to be a highly effective probe of the optical-UV EBL.

Subject headings: gamma rays: observations - intergalactic medium - galaxies: active - instrumentation: detectors

\section{Introduction}

In the last few years the study of galaxy formation and evolution has seen tremendous progress. Instruments at many different wavelengths have begun to penetrate to the relevant redshifts. One important prediction of models of galaxy formation and evolution is the nature of the radiation field produced by star formation. One way to probe the resulting extragalactic background light (EBL) is to measure the attenuation through pair production of gamma rays from distant sources. However, without a large sample of sources distributed across a wide redshift range, it is difficult to distinguish between extragalactic absorption and characteristics peculiar to individual sources. The Large Area Telescope (LAT) instrument on The Gamma-Ray Large Area Space Telescope (GLAST) will observe gamma rays with energies from $20 \mathrm{MeV}$ to $>300 \mathrm{GeV}$. The GLAST LAT will be the first instrument able to probe the intergalactic radiation field by observing the absorption of gamma rays from a large number of extragalactic point sources as a function of redshift over a wide range. Groundbased telescopes can measure the attenuation of $\mathrm{TeV}$ emission by intergalactic IR radiation (Stecker, DeJager, \& Salamon 1992; Macminn \& Primack 1996; Madau \& Phinney 1996). However, these telescopes will measure the spectra of relatively small number of sources, making it more difficult to resolve the question of whether differences between sources are due to intergalactic attenuation or intrinsic peculiarities. Furthermore, the high pair production opacity of the IR radiation limits TeV probes of the EBL to a narrow, low-redshift range. GLAST, on the other hand, will observe thousands of sources, and will measure less drastic attenuation of $\mathrm{GeV}$ photons by optical and UV radiation. The energy range and capabilities of GLAST are thus ideal for probing the EBL to cosmological distances.

This paper reports our first modeling of the ability of GLAST to measure the extragalactic background light absorption. In order to do this, we need 1) models of the intergalactic radiation field, 2) the luminosity function of extragalactic gamma-ray sources, and 3) parameters of the instrument. In Section 2.1 we briefly review models for the intergalactic radiation field and the resulting gamma-ray opacity as a function of redshift. In Section 2.2 , we describe the two gamma-ray blazar luminosity functions used. In Section 2.3 we 
describe the parameters used to simulate GLAST. In Section 3 we discuss the simulation procedure, including the two different models of blazar input spectra and the two models for the intergalactic radiation field. In Section 4 we present our results and conclusions.

\section{Framework}

\subsection{Extragalactic Background Light}

Gamma rays with $E>10 \mathrm{GeV}$ traveling through intergalactic space will interact through pair production with the extragalactic background starlight (EBL) emitted by galaxies. The total center-of-mass energy must be high enough to produce the electron-positron pair, and, for a wide range of EBL models, the attenuation becomes significant only above $\sim 10 \mathrm{GeV}$. The cross section is maximized when the EBL photon energy $\epsilon_{E B L} \sim \frac{1}{2}\left(\frac{1000 \mathrm{GeV}}{E_{\gamma}}\right)$ $\mathrm{eV}$, with $E_{\gamma}$ in $\mathrm{GeV}$ (Stecker, DeJager \& Salamon 1992). For $10 \mathrm{GeV}$ to $\mathrm{TeV}$ gamma rays this corresponds to $\epsilon_{E B L}$ in the optical-UV range. Salamon \& Stecker (1998) calculated the opacity of high energy gamma-rays to redshift $z=3$. To estimate the stellar emissivity and spectral energy distributions vs. redshift they adapted the analysis of Fall, Charlot, \& Pei (1996), consistent with the Canada-France Redshift Survey, and included corrections for metallicity evolution. They found that the stellar emissivity peaks between $z=1$ and 2 before falling off, leading to a significant redshift-dependent absorption below $z=3$. Other models, e.g. by Primack et al. (1999), provide for significant attenuation at even larger redshifts. More recently, Bernstein, Freedman, \& Madore (2002a,b) have made the first direct measurement of the optical-UV EBL integrated over redshift. As shown in Section 3, our technique is a powerful discriminator among models, giving information about the era of galaxy formation and evolution.

\subsection{Gamma-ray Blazars}

\subsubsection{Blazar Luminosity Function}

The Energetic Gamma Ray Experiment Telescope (EGRET) detected more than 60 blazar-type quasars (Mukherjee et al. 1997) emitting gamma rays with $E>100 \mathrm{MeV}$. These sources are flat-spectrum radio-loud quasars (FSRQs) and BL Lac objects, often exhibiting non-thermal radio continuum spectra, violent optical variability and/or high optical polarization. They are also highly variable and powerful gamma-ray sources. The EGRET blazars whose optical redshifts have been measured lie between $z=0.03$ and 2.28. The 
redshift distribution is consistent with the observed distribution of FSRQs, which extends up to $z=3.8$. However, since the luminosity function determines the statistical power of our technique versus redshift, and since this function is still relatively unconstrained, we use two different models for the blazar luminosity function.

The first model, by Salamon \& Stecker (1996), makes the assumption that blazars seen in gamma-rays above $100 \mathrm{MeV}$ are also seen in the radio as FSRQs. This model assumes that the gamma and radio ray luminosity functions are linearly related as

$$
\rho_{\gamma}\left(L_{\gamma}, z\right)=\eta \rho_{r}\left(L_{r}, z\right)
$$

where $\eta$ is a parameter of the model and

$$
\rho_{r}\left(L_{r}, z\right)=10^{-8.15}\left\{\left(\frac{L_{r}}{L_{c}(z)}\right)^{0.83}+\left(\frac{L_{r}}{L_{c}(z)}\right)^{1.96}\right\}^{-1}
$$

with $\log _{10} L_{c}(z)=25.26+1.18 z-0.28 z^{2}$. The units of the comoving density $\rho$ are $\mathrm{Mpc}^{-3} \times$ ( unit interval of $\left.\log _{10} L\right)^{-1}$ and the units of $L$ are $\mathrm{W} \mathrm{Hz}^{-1} \mathrm{sr}^{-1}$. Using cosmological parameters $\Omega_{M}=1, \Omega_{\Lambda}=0$, and $H_{0}=50 \mathrm{~km} / \mathrm{s} / \mathrm{Mpc}$, the model is constrained to predict the number of blazars observed by EGRET.

The number of sources with redshift in the interval $z+\Delta z$ seen at Earth with a flux for $E>100 \mathrm{MeV}$ in the interval $F+\Delta F$ is given by (Salamon \& Stecker 1996)

$$
\frac{d N}{d F d z} \Delta z \Delta F=4 \pi R_{0}^{3} r^{2} \Delta r \rho_{\gamma} \Delta\left(\log _{10} L\right)
$$

with $R_{0} r=\frac{2 c}{H_{0}}\left(1-(1+z)^{-1 / 2}\right)$, where $H_{0}$ is the Hubble expansion rate. Combining the choice of parameters given by Salamon \& Stecker (1996) with a GLAST flux sensitivity of $1.5 \times 10^{-9}$ photons $\mathrm{cm}^{-2} \mathrm{~s}^{-1}$, a number of $\sim 9000$ blazars is expected to be observed with redshifts up to $z \sim 4$.

The second model, by Chiang \& Mukherjee (1998), does not assume a correlation between luminosities at gamma ray energies and at other wavelengths. This model parametrizes the luminosity function as

$$
\begin{array}{cl}
\frac{d N}{d L_{0} d V} \propto\left(\frac{L_{0}}{L_{B}}\right)^{-\gamma_{1}}, & L_{0} \leq L_{B} \\
\frac{d N}{d L_{0} d V} \propto\left(\frac{L_{0}}{L_{B}}\right)^{-2.2}, & L_{0}>L_{B}
\end{array}
$$

with de-evolved luminosity $L_{0}=L /(1+z)^{\beta}$ and a maximum cutoff redshift of $z_{\max }=5$. The energy range of this integrated luminosity is $E>100 \mathrm{MeV}$. The best fit found for 
this broken power law is parametrized by $\gamma_{1} \lesssim 1.2, L_{B}=1.1 \times 10^{46} \mathrm{erg} / \mathrm{s}$ and $\beta=2.7$, with cosmological parameters $\Omega_{M}=1, \Omega_{\Lambda}=0$, and $H_{0}=75 \mathrm{~km} / \mathrm{s} / \mathrm{Mpc}$. Each model was separately fit in a self-consistent fashion to the EGRET data to produce the luminosity functions. More recent cosmological data suggest a non-zero value for $\Omega_{\Lambda}$. The impact on the luminosity function, however, is small; we therefore retain the original model, along with the fit to the data, for our calculations. Of course, one of the important goals of the GLAST mission will be to constrain the luminosity function.

\subsubsection{Blazar Spectra}

The spectra of the blazars observed by EGRET are well characterized in the $E>$ $100 \mathrm{MeV}$ range by power laws with an average photon spectral index of $-2.15 \pm 0.04$ (Mukherjee et al. 1997). The spectra of some individual blazars have a measured index significantly different from the mean value, suggesting true scatter in the distribution of blazar spectra, which our simulation takes into account as described below. More importantly, most of the EGRET blazars have not been detected by TeV telescopes; for many of these sources, this implies a spectral break or rolloff at some energy between the EGRET and TeV energy ranges. Intergalactic attenuation, the very effect explored in this paper, would account for the lack of detection of high-redshift objects, but there are relatively low-redshift blazars that are bright in the EGRET range and undetected in the TeV range. More tellingly, most of the TeV blazars belong to the same subset of blazars, the X-ray selected BL Lac objects (XBLs). Since only a small fraction of the EGRET blazars are XBLs, this implies that the non-XBL blazars may have spectra with intrinsic rolloffs independent of any intergalactic attenuation effects. Finally, blazars that have been detected in both the GeV and TeV ranges have TeV fluxes that are lower than simple extrapolations of the EGRET power laws would suggest. Of course, such an extrapolation over such a wide range of energies is unreasonable. Most of the models for blazar spectra attribute both the $\mathrm{GeV}$ and $\mathrm{TeV}$ emission to the same inverse Compton component of the emission. However, with little observational data in the 30-300 GeV range, no firm conclusions can be drawn about the precise shape of the spectra. Indeed, this is one of the motivations for the next generation of experiments.

Our technique, as described in Section 3, is to form the ratio of the observed fluxes for $E>10 \mathrm{GeV}$ and $E>1 \mathrm{GeV}$,

$$
\frac{F(E>10 \mathrm{GeV})}{F(E>1 \mathrm{GeV})}
$$

This ratio is simple, robust, and insensitive to rolloffs above $\sim 50 \mathrm{GeV}$ for most EBL models as shown in Section 3.1. We attempt to bracket the range of possible spectra by first analyzing a sample of blazars whose power law spectral indices are normally distributed 
around a mean of -2.15 with standard deviation 0.04 , representing a situation where there is a range of spectral indices but no intrinsic rolloff in this energy range. To model intrinsic rolloffs, we then repeat the analysis with a sample of blazars whose unredshifted spectra have a broken power law with mean index -2.15 below $50 \mathrm{GeV}$ and -3.15 above, again with a standard deviation of 0.04 in each case.

\subsection{GLAST}

The Gamma-ray Large Area Space Telescope (GLAST) is under development with a planned launch in 2006 (Michelson 2001). The Large Area Telescope (LAT) of GLAST will observe gamma rays with energies from $20 \mathrm{MeV}$ to $>300 \mathrm{GeV}$. GLAST will have a much larger effective area than EGRET, especially at higher energies (peak effective area $>8000$ $\mathrm{cm}^{2}$ at $>1 \mathrm{GeV}$ ), a larger field of view, and sub-arcminute scale source localization. GLAST should be able to reach a $5 \sigma$ point source flux sensitivity of less than $1.5 \times 10^{-9}$ photons $\mathrm{cm}^{-2}$ $\mathrm{s}^{-1}$ for $E>100 \mathrm{MeV}$ within five years. As noted above, using the distribution of blazars observed by EGRET and extrapolating to lower fluxes, it is estimated that GLAST will detect thousands of blazars. Improved angular resolution should allow a high percentage of optical identifications and redshift measurements, depending on the available ground-based resources. Improved high-energy performance should yield accurate flux determinations above $10 \mathrm{GeV}$ for many of these sources. Note that our modeling is based on the generic parameters outlined in the GLAST Science Requirements Document (Michelson 2001); the performance of the flight instrument may be substantially better.

\section{Procedure}

To simulate the gamma-ray sources observable by GLAST, we need a reasonable extrapolation of the EGRET source distribution to the GLAST flux limit. We used the two luminosity functions described in section 2.2.1 for this purpose, but our main conclusions do not depend significantly on this choice. We note that any predictions made now will be supplanted by the data GLAST itself provides.

Before any observational selection, according to the luminosity function by Salamon \& Stecker (1996), 12,000 blazars in principle will have fluxes in the range detectable by GLAST. Each one was assigned a random luminosity and redshift according to this model.

With the luminosity function by Chiang \& Mukherjee (1998) we generated 10,000 blazars, between redshifts 0 and 5 according to Figure 6 of their paper. 
For both samples, the flux of each blazar was then calculated according to

$$
F=L \frac{(1+z)^{2-\alpha}}{4 \pi d_{l}^{2}(z)}
$$

where $\alpha$ is the photon spectral index and $d_{l}$ is the cosmological luminosity distance $d_{l}=$ $\frac{2 c}{H_{0}}(1+z)\left[1-(1+z)^{-1 / 2}\right]$.

Only blazars with observed flux greater than $1.5 \times 10^{-9}$ photons $\mathrm{cm}^{-2} \mathrm{~s}^{-1}$ for $E>$ $100 \mathrm{MeV}$ are allowed in the sample. The $E>10 \mathrm{GeV}$ flux of each blazar was calculated by adding two effects. First, each blazar was given a random, normally distributed spectral index, $-2.15 \pm 0.04$. An index of -2.15 yields a flux ratio $\frac{F(E>10 \mathrm{GeV})}{F(E>1 \mathrm{GeV})}$ of $\sim 0.07$. Also included was the redshift-dependent absorption above $10 \mathrm{GeV}$. The form of the dependence was parameterized from Figure 6 of Salamon \& Stecker (1998), with metallicity corrections. In this EBL model, $\Omega_{M}=1, \Omega_{\Lambda}=0$, and the value of $H_{0}$ scales out. We set the absorption for $z>3$ for this model equal to the absorption at $z=3$, both because it is a conservative assumption and because it is physically plausible (little stellar emissivity, and smaller scale and path lengths, for $z>3$ ). To produce observed fluxes from these intrinsic fluxes, each blazar was assigned a random position on the sky and, assuming an exposure equivalent to two full years, Galactic and extragalactic backgrounds were added. The Galactic backgrounds were derived from the diffuse model used in EGRET analysis (Hunter et al. 1997). To take into account the extragalactic background, we added a second, fixed, background component, with intensity $4 \times 10^{-6}$ photons $\mathrm{cm}^{-2} \mathrm{~s}^{-1} \mathrm{sr}^{-1}$ for $E>100 \mathrm{MeV}$ and a power law index of -2.15 , under the assumption that GLAST may resolve a significant fraction of the EGRET isotropic background (Stecker \& Salamon 1999). This component represents the sum of the flux from unresolved blazars and any truly diffuse background contribution. Any blazar within $10^{\circ}$ of the Galactic plane and any blazar whose observed flux was less than $5 \sigma$ above the background flux at $E>1 \mathrm{GeV}$ was removed from the sample, leaving $\sim 9100$ blazars (Stecker \& Salamon 1996) or 8200 blazars (Chiang \& Mukherjee 1998). Figure 1 shows a histogram of the number of blazars in each 0.5 redshift bin. The model by Chiang \& Mukherjee (1998) predicts a population of blazars that are intrinsically brighter when compared to the model by Stecker \& Salamon (1996). In that case, GLAST would detect more blazars at higher redshift as can be observed from the graph. We note that, with no EBL attenuation, for $z>3$ and requiring more than 5 detected photons $(E>10 \mathrm{GeV})$, GLAST would see $\sim 60$ blazars using the Stecker \& Salamon luminosity function, or $\sim 700$ blazars using the Chiang \& Mukherjee luminosity function. 


\subsection{Calculating the flux ratios}

The integrated fluxes of each blazar for $E>1 \mathrm{GeV}$ and $E>10 \mathrm{GeV}$ were used to generate observed fluxes using Poisson distributions equivalent to two full years of exposure. For each blazar, we calculated the ratio between these fluxes. The error in each flux ratio

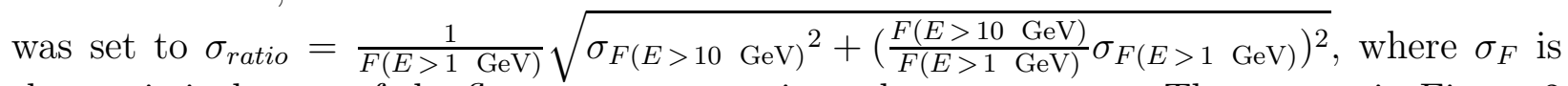
the statistical error of the flux measurement in each energy range. The crosses in Figure 2 show the weighted mean ratio in each redshift bin. To avoid the bias of small number Poisson statistics toward lower values, the flux ratio of each source was weighted by the Poisson error of the $E>1 \mathrm{GeV}$ flux, rather than the formal, propagated error of the flux ratio. The diamonds show the same ratio when the intergalactic absorption is removed from the observed blazar fluxes. In all cases the error bars are statistical, obtained by computing the rms scatter within each redshift bin and dividing by $\sqrt{N}$. The analytically derived flux ratio using the opacity model of Salamon \& Stecker is plotted as a solid curve. For comparison, the dashed lines in Figure 2 show the same results with no intergalactic absorption.

We repeated the entire analysis with the blazar spectra changed from single power laws with mean index -2.15 to broken power laws with mean index -2.15 below $50 \mathrm{GeV}$ (at the source) and -3.15 above. The results are plotted as crosses in Figure 3. Although fewer blazars have detected flux above $10 \mathrm{GeV}$, the effects of absorption are still apparent. Note that sources with no detectable flux above $10 \mathrm{GeV}$ (zero photons) still provide important information; indeed, neglecting them introduces a bias. The modified $\chi^{2}$ statistic used here (Mighell 1999) accounts for these sources.

The ratio obtained without EBL absorption is presented as diamonds, along with the analytically derived flux ratio (dashed line). As can be easily seen, this flux ratio is not constant as a function of redshift. This is a consequence of defining the break in the index for a given energy at the source.

\subsection{Other EBL models}

Primack and collaborators combined theoretical modeling with observational data to develop semi-analytic models of galaxy formation and evolution (Primack et al. 1999). Their models permit a physical treatment of the processes of galaxy formation and evolution in a cosmological framework, including gravitational collapse, mergers, etc., rather than relying on pure luminosity evolution of the galaxies existing today. We use their calculations of opacities to gamma rays at redshifts up to $z=5$. The cosmological parameters used are $\Omega_{M}=0.4, \Omega_{\Lambda}=0.6$, and $H_{0}=60 \mathrm{~km} / \mathrm{s} / \mathrm{Mpc}$. The luminosity functions use a different value 
for $H_{0}$, but for our purposes this difference does not significantly affect the results; as shown by Blanch and Martinez (2003), the gamma-ray horizon has a relatively weak dependence on $H_{0}$. Note that these opacities, with their different cosmological parameter sets, should not be thought of as predictions, but rather as another set of reasonable values to illustrate the discriminating power of our technique. The results are shown as triangles in Figure 2 and Figure 3, along with the lines representing the analytical prediction. The fact that the flux seems to be more highly attenuated is not important. What is more interesting is that the decrease in flux ratio from $z=2.5$ to $z=5$ is observable. This indicates, assuming the availability of gamma-ray sources and sufficient EBL density, that EBL absorption can effectively probe galaxy formation at those redshifts, a regime of intense theoretical interest. More recently, Oh (2001) performed an independent calculation of the opacity of gammaray blazar emission to pair production by UV photons as a function of redshift. While not addressing the detectability of high-redshift blazars by GLAST in detail, he obtains attenuation factors that vary strongly with redshift in a manner roughly consistent with the calculations we have used.

\section{Results and Conclusions}

Extragalactic attenuation of gamma-rays by low-energy background photons produces a distortion in the spectra of gamma-ray blazars that increases with increasing redshift. Because we cannot distinguish the difference between extragalactic attenuation and intrinsic effects in individual blazar spectra, statistical analysis of a large sample of blazars such as those presented in this paper is a powerful tool to study EBL absorption. Although AGILE, the next GeV mission (Tavani et al. 2001), will produce a significant increase in the total number of blazars and therefore refine the blazar luminosity function and evolution, GLAST will be the first mission to observe a large sample of high redshift blazars with sufficient statistics to separate intrinsic differences between blazars from redshift dependence of EBL absorption. Our results indicate that the redshift dependence of the attenuation should be easily detectable by GLAST even when the diffuse background is taken into account and possible high-energy intrinsic rolloffs are considered.

Selection effects, both from GLAST itself and from optical coverage of redshift determinations, will primarily affect sources with low flux. These sources will have poorly measured flux ratios, and will suffer from optical selection effects due to their more poorly determined positions. Other biases include the locations of optical telescopes, source clustering, and other effects. It will be important to catalog these effects explicitly; in particular, insuring adequate optical coverage may require active preparation and participation. 
GLAST will be able to measure the differences in blazar attenuation in the cosmologically interesting range in redshift from $z=1$ up to $z=5$. This is in contrast to ground-based observations of $\mathrm{TeV}$ attenuation by IR radiation, which will only be able to measure differences well below $z=1$, where the IR becomes opaque. As the energy threshold of the ground based experiments drops over time, their redshift range will increase, but will remain limited to low redshifts except for exceptional, statistically insignificant special cases, especially given their generally small fields of view. More than establishing that EBL attenuation occurs, GLAST will be able to distinguish between different EBL models. This would validate EBL attenuation as a direct cosmological probe.

We emphasize that this analysis will require redshift determinations of a large fraction of GLAST blazars. This is another example of the importance of cross-wavelength studies: by using optical measurements of blazar redshifts, gamma-ray measurements can uniquely probe the optical-UV EBL. A redshift measurement for thousands of high-redshift sources is not a trivial undertaking, but the effort will be well rewarded.

Even after observation of a redshift-dependent effect, the possibility would remain that the spectral evolution of gamma-ray blazars might coincidentally mimic redshift-dependent EBL absorption. For example, if blazars that formed in the early universe suffered more internal attenuation than blazars that formed later, the same effect could be produced. Note that blazars are variable, and there are some indications that their spectra can become harder when they flare (Sreekumar et al. 1996). Evolution in flaring probability could produce the same effect as actual spectral evolution from a statistical standpoint (for example, a higher percentage of high-redshift blazars might be observed in a quiescent phase), although one would expect the GLAST flux limit to produce a selection effect in the opposite direction. In any case, observation of a redshift-dependent spectral softening will provide an important constraint. Theorists will have to decide the likelihood of an evolutionary conspiracy.

We acknowledge useful conversations with Bill Atwood, who first suggested using the large statistics of GLAST AGNs to look for systematic effects of extragalactic background light attenuation with redshift. We would also like to thank David Thompson, Seth Digel, Floyd Stecker, and Mike Salamon for their useful comments, and James Bullock and Joel Primack for providing calculations from their models. We would like to thank the referees for many useful comments, in particular drawing our attention to the Blanch \& Martinez article on cosmological effects in emissivity evolution. 


\section{REFERENCES}

Bernstein, R.A., Freedman, W.L., \& Madore, B.F. 2002a, ApJ, 571, 56

Bernstein, R.A., Freedman, W.L., \& Madore, B.F. 2002b, ApJ, 571, 107

Blanch, O., \& Martinez, M. 2001, astro-ph/0107582

Chiang, J., \& Mukherjee, R. 1998, ApJ, 496, 752

Chiang, J., et al. 1997, ApJ, 452, 156

Hunter, S.D., et al. 1997, ApJ, 481, 205

Macminn, D. \& Primack, J.R. 1996, Space Sci. Rev. 75, 413

Madau, P. \& Phinney, E.S. 1996, ApJ, 456, 124

Michelson, P. 2001, in AIP Conf. Proc. 587, $\gamma$-ray Astrophysics, eds. S. Ritz, N. Gehrels \& C.R. Schrader, (Melville, N.Y.: AIP), 713, http://glast.gsfc.nasa.gov/SRD

Mighell, K.J. 1999, ApJ, 518, 380

Mukherjee, R., et al. 1997, ApJ, 490, 116

Oh, S.P. 2001, ApJ, 553, 25

Primack, J.R., Bullock, J.S., Somerville, R.S., MacMinn, D. 1999, APh, 11, 93

Salamon, M.H., \& Stecker, F.W. 1998, ApJ, 493, 547

Sreekumar, P. et al. 1996, ApJ, 464, 628

Stecker, F.W., \& Salamon, M.H. 1999, Astrophys. Space Sci. 261, 327

Stecker, F.W., \& Salamon, M.H. 1996, ApJ, 464, 600

Stecker, F.W., DeJager, O.C. \& Salamon, M.H. 1992, ApJ, 390, L49

Tavani, M., et al. 2001, in AIP Conf. Proc. 587, $\gamma$-ray Astrophysics, eds. S. Ritz, N. Gehrels \& C.R. Schrader, (Melville, N.Y.: AIP), 729 
Fig. 1.- Number of detectable blazars in each redshift interval. The solid curve is the population according to Stecker \& Salamon (1996), the dotted line corresponds to the model by Chiang \& Mukherjee (1998). The spectra of the blazars are power laws with index $-2.15 \pm 0.04$.

Fig. 2.- Mean observed flux ratio, as described in the text, using the luminosity function by Salomon \& Stecker (a) and Chiang \& Mukherjee (b). Each cross is the mean observed flux ratio in the corresponding redshift interval with fluxes attenuated by the EBL of Salamon \& Stecker. The solid curve is the ratio calculated with the same model. The triangles and the dash-dot-dotted line are the mean observed and calculated flux ratios for blazars, with the EBL attenuation model of Primack et al. Finally, the diamonds show the mean observed flux ratio with no EBL attenuation and the dashed line is the corresponding calculated ratio.

Fig. 3.- Mean observed flux ratio as described in the text for blazars with broken spectral index at $50 \mathrm{GeV}$ at the source. The luminosity function is obtained from Stecker \& Salamon (a) and Chiang \& Mukherjee (b). The EBL attenuation is given by either Salamon \& Stecker (crosses) or Primack et al (triangles). The analytically calculated flux ratios for each luminosity function are shown by the solid and dash-dot-dotted lines respectively. The dashed line and the diamonds show the same results when there is no EBL attenuation. 


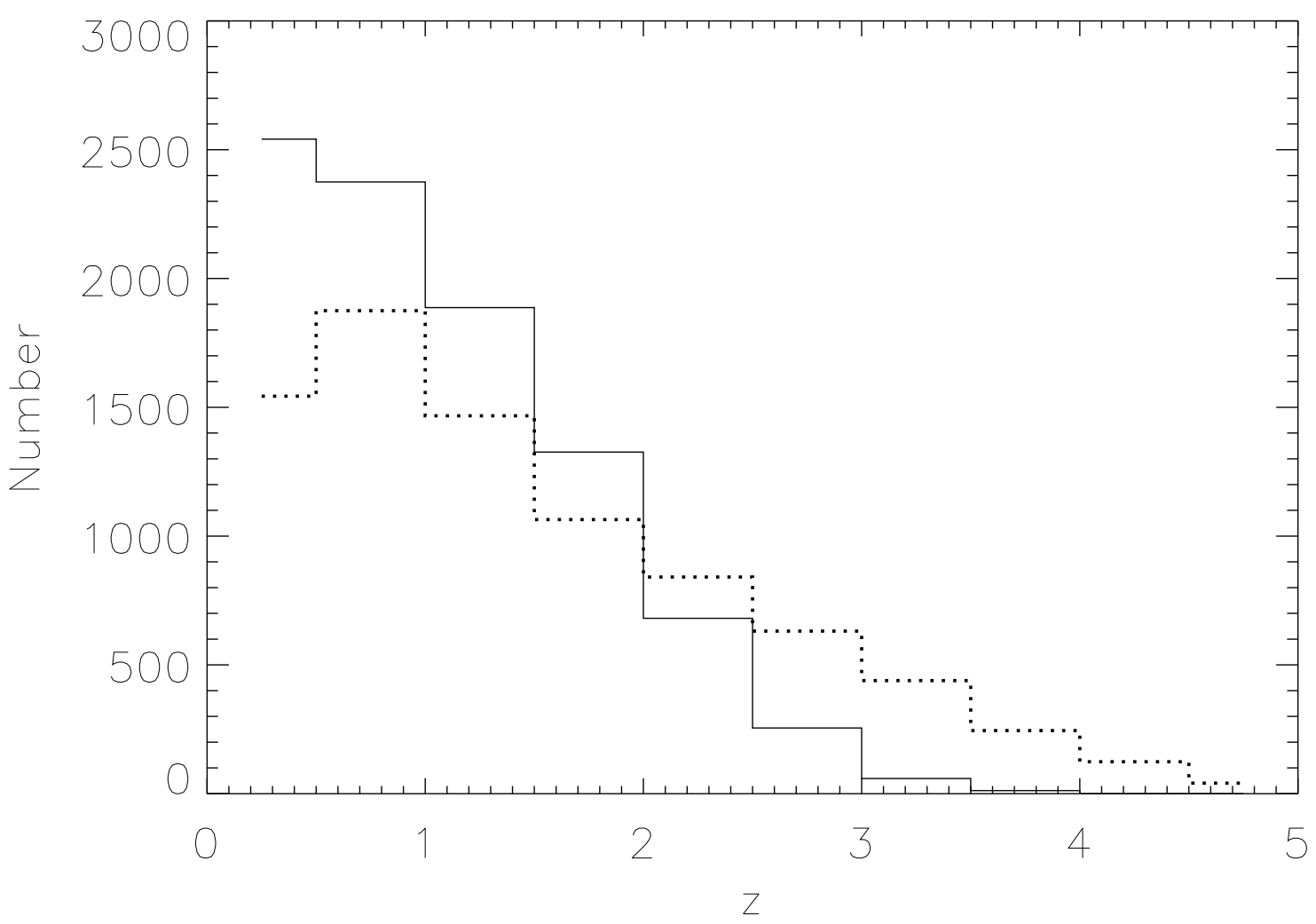



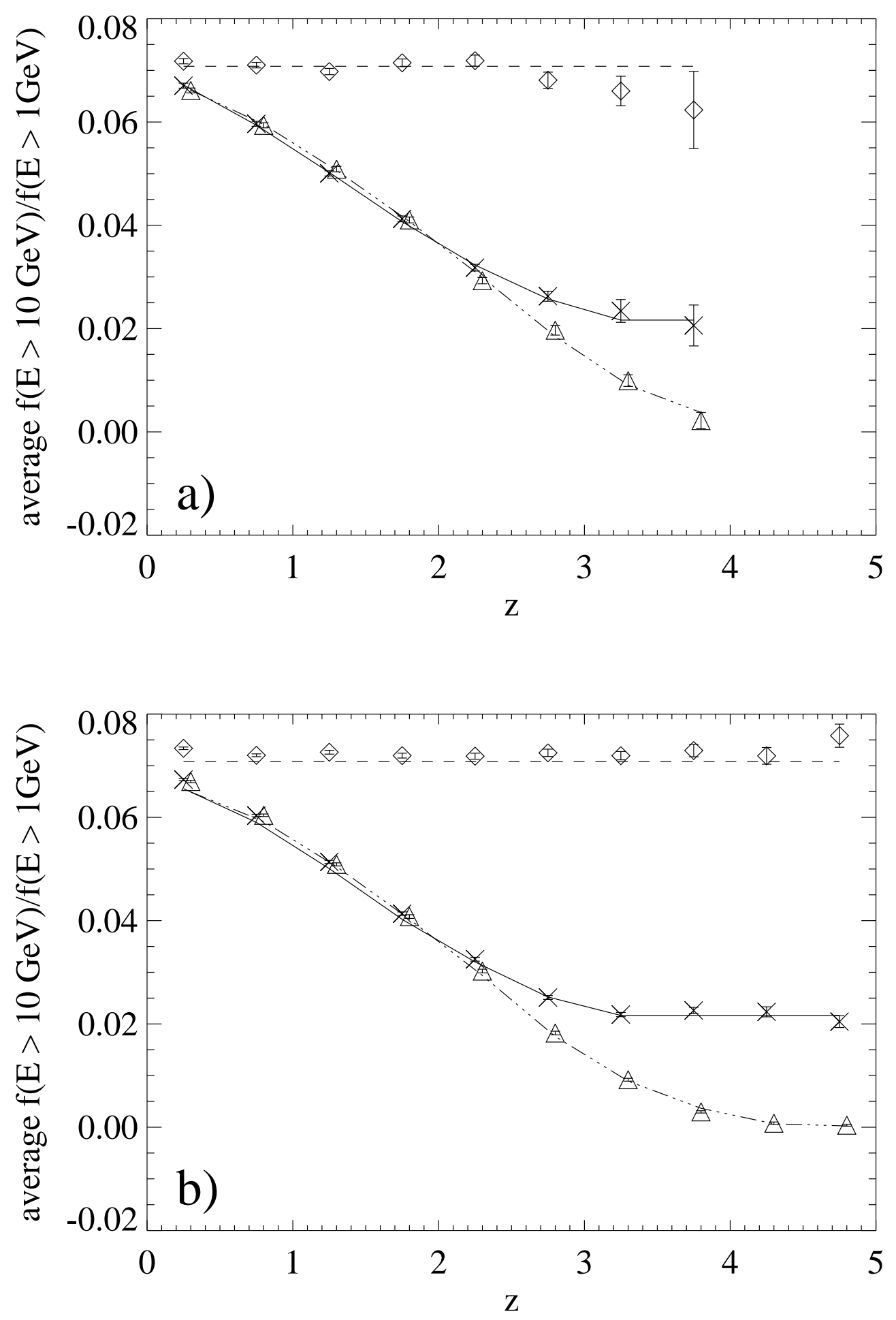

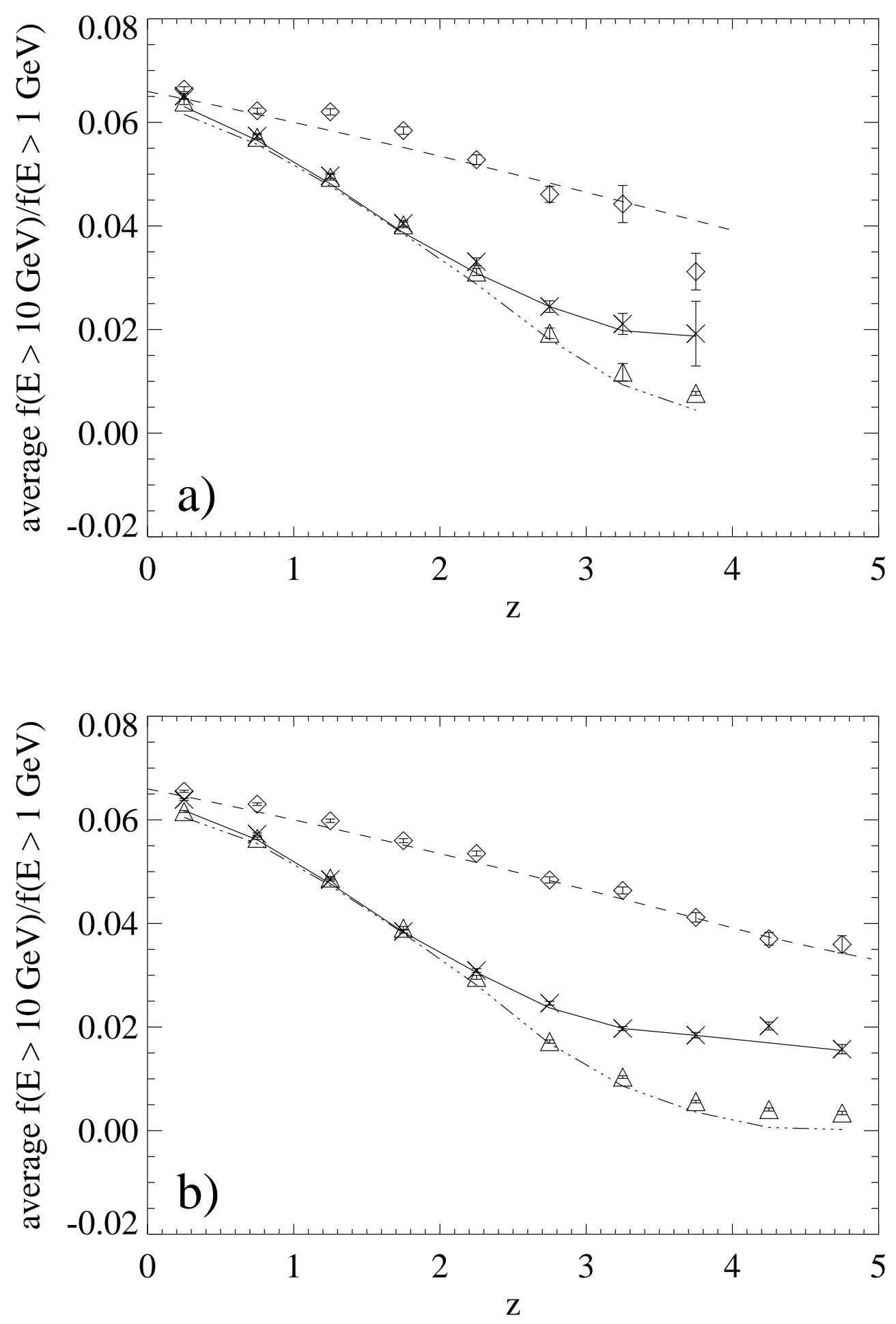